\title{
The science of education reform
}

\author{
Much cognitive neuroscience has focused on determining how to improve learning. This research has promise to \\ guide effective education reform, but future translation efforts must be evaluated as rigorously as basic science.
}

$\mathbf{T}$ his September, the National Research Council of the National Academy of Sciences released a report concluding that despite many years of focused standards-based reform, improvements in United States science education have been minimal. Another study reports that a large achievement gap remains between economically disadvantaged students and their wealthier peers. These deficiencies are all the more significant in light of the federal government's No Child Left Behind Act, which mandates that each school district be held accountable for pupils' achievements, and that all states meet certain proficiency standards.

Cognitive neuroscientists have made a substantial effort to understand how the brain learns and adapts, and there has been considerable interest in translating this work into practice. In 2004, the National Science Foundation (NSF) announced grants totaling over $\$ 90$ million to establish multidisciplinary 'Science of Learning Centers,' collaborations between scientists and educators to devise practical and scientifically based teaching methods that take cutting-edge scientific discoveries into account. However, this program has yet to yield tangible benefits for teachers. Instead, the NSF center at Dartmouth College in Hanover, New Hampshire (which had the strongest neuroscience component of all the centers) is closing up its program prematurely; Michael Gazzaniga, one of the scientists involved in this effort, says that one problem was a disconnect between what NSF administrators wanted and what the scientists were able to deliver, as the administrators expected tangible results too soon.

There is little doubt that neuroscientists have made strides in understanding how the young brain learns. For example, young readers depend on the left posterior superior temporal cortex, an area implicated in phonological skill acquisition, and as literacy is acquired, the visual word form area in the left occipital temporal region becomes activated. Dyslexics, however, show abnormal activation of the right parietal cortex ${ }^{1}$. Likewise, parietal brain areas are involved in representing both physical size and numerosity ${ }^{2}$.

It is not yet clear, however, how we should apply such findings to education in the classroom. Brian Wandell of Stanford University likens the situation to the genomics revolution, which was predicted to dramatically alter the state of medicine and its delivery a decade ago, but which has yet to deliver fully on this promise. Like genomics, insights from cognitive neuroscience should alter the educational landscape, but the field is not there yet. Usha Goswami at the University of Cambridge notes that what neuroscience can currently offer is the possibility of identifying neural indices of a child's potential difficulties, and the framework to scientifically test neural hypotheses that may influence educational practices ${ }^{1}$.

The public clearly wants information about how the brain learns and is eager to embrace any 'magic bullet' that is even remotely associated with science. This September, Durham county in the United Kingdom announced that it would give 5,000 pupils daily doses of fish-oil supplements to improve their exam scores, quoting trials (with no control group) showing that fish-oil supplements had improved the concentration and learning abilities of young children (http://news.bbc. co.uk/1/england/5318930.stm). Similarly, many state schools in the UK embraced a program called Brain Gym, which claims that a series of simple physical movements will "integrate all brain areas and promote efficient communication among the many nerve cells" (http://www. guardian.co.uk/life/badscience/story/0,1733683,00.html).

Although programs based on faulty science such as Brain Gym abound, there are few examples of translation from the laboratory to the classroom that have been rigorously reviewed. A few years ago, almost a quarter of a million schoolchildren in public schools in Philadelphia participated in a training program aimed at ameliorating reading difficulties ${ }^{3}$. The software involved, marketed by Scientific Learning Corporation, is based on neuroscience research showing that auditory processing defects can underlie dyslexia. However, to date, no independent review has corroborated the validity of this program in the classroom, and it is unclear how this approach compares with other strategies aimed at raising reading scores.

Scientists have basked in public trust, and we must take care not to abuse this privilege. Practical applications are a key outcome of research, and we applaud efforts to translate cognitive neuroscience into practice. However, better evaluation of these applications is essential. Drug companies must pass an extensive regulatory process before their medications are approved, yet behavioral remedies and training programs seemingly require little, if any, review before they are unleashed on unsuspecting children and teachers. The field is in need of an honest broker to evaluate these programs and judge their efficacy and cost-effectiveness. Scientists should be active participants in ensuring that all translational efforts are reviewed as rigorously as the basic science findings.

At the same time, it is critical to recognize that scientists are not the ultimate experts on education. Translational efforts should be guided by determining what problems teachers currently face in the classroom, and should be evaluated based in part on their experience of what works. Scientists should embrace any opportunities for dialogue with educators, encouraging them to be skeptics and advocating a culture in which administrators and teachers scientifically evaluate products before accepting them. Bringing the expertise of cognitive neuroscience into our schools may still be a pipe dream for now, but we must take care not to erode public trust before the promise becomes a reality.

1. Goswami, U. Nat. Rev. Neurosci. 7, 406-411 (2006).

2. Feigenson, L., Dehaene, S. \& Spelke, E. Trends Cogn. Sci. 8, 307-314 (2004).

3. Nat. Neurosci. 7, 1 (2004).

View more background material on Connotea at http://connotea.org/user/ NatNeurosci/tag/editorial200611. 